\title{
Environmentally controlled dimorphic cycle in a fission yeast
}

\author{
M. Sipiczki, ${ }^{1,2,3}$ K. Takeo, ${ }^{3}$ M. Yamaguchi, ${ }^{3}$ S. Yoshida ${ }^{3}$ and I. Miklos ${ }^{1}$
}

Author for correspondence: M. Sipiczki. Tel: +36 52316 666. Fax: +3652348550.

e-mail : lipovy@tigris.klte.hu

1,2 Department of Genetics ${ }^{1}$ and Institute of Biology2, University of Debrecen, P.O. Box 56, H-4010 Debrecen, Hungary

3 Division of Ultrastructure and Function, Research Center for Pathogenic Fungi and Microbial Toxicoses, Chiba University, Chiba, Japan

\begin{abstract}
The fission yeast Schizosaccharomyces pombe shows bipolar growth and is a convenient model for studying cell polarity and polar growth. This paper shows that the related Schiz. japonicus var. japonicus can switch to unipolar growth and can exist in both yeast and mycelial phases. On solid media, the yeast phase is unstable and prone to switch to the mycelial form, which shows unipolar growth by tip elongation. The hyphae can colonize the body of the substrate (true mycelium) or just its surface (pseudo-mycelium). The yeast-tomycelium transition and the growth of the mycelium are regulated by a nutritional gradient and are associated with extensive vacuolation. The mycelium can convert into arthroconidia or return to the yeast phase in response to environmental changes. These environmentally controlled morphological transitions make Schiz. japonicus var. japonicus an attractive model for the investigation of cell polarity and morphogenesis.
\end{abstract}

Keywords: Schizosaccharomyces, polar growth, dimorphic transition, vacuolation, arthrospores

\section{INTRODUCTION}

Although cell polarity and polar growth is a phenomenon exhibited throughout the eukaryotic world, the mechanisms which control polarity remain poorly understood. Because of the strict polarity and highly regular mode of division of its cells, the unicellular ascomycete Schizosaccharomyces pombe has proved to be an excellent model for studying the phenomenon of cell polarity. The cells show cylindrical morphology with two poles in the exponential phase of growth. They grow by polar extension and divide by medial septation (for reviews see Johnson et al., 1982; Snell \& Nurse, 1993). The cells with walls newly formed in the previous cell cycle start growing first at the 'old end' (the end that existed in the previous cycle as the end of the mother cell), but cells with old walls produced in earlier cell cycles may start growing at the 'new end' or at both ends simultaneously (Miyata et al., 1986). In liquid media, nearly all cells show more growth at the old end (Mitchison \& Nurse, 1985; May \& Mitchison, 1995), whereas on agar pads, significant percentages of cells show more extension at the new end or equal growth at both ends (Miyata et al., 1986, 1990; Sipiczki et al., 1993).

The cytoplasmic cytoskeleton of the growing cells is also polarized. Most of the actin shows a polar location when the cell grows (Marks \& Hyams, 1985) and the cytoplasmic microtubules run from pole to pole (Hagan $\&$ Hyams, 1988). At the onset of mitosis, these microtubules disappear, and a new cytoplasmic array is formed in each daughter cell by the microtubuleorganizing centres (Hagan \& Hyams, 1988). These centres are located near the site of cell division ('new end'), so most of the newly formed microtubules arising from them are directed towards the opposite ('old') end of the cell. It is not yet clear whether this polarity of the new array determines the polarity of the cell and the site of growth initiation in the new cell cycle. Nevertheless, the growth morphology of the filamentous mutant sep11 suggests that the establishment of cell polarity and the site of growth also require communication between the cytoplasmic cytoskeleton and the cell wall. The cells of this mutant do not separate after septation so their ends remain covered by unsplit septa. These septa prevent the cell from growing at its ends and make it commence growth at subapical lateral sites (Sipiczki et al., 1993). This results in a torsion of the linearity of the cell axis, but does not affect the overall polarity or the oldend/new-end growth patterns (Sipiczki \& Grallert, 1997).

To obtain further insight into the determination of cell polarity in fission yeast cells, we initiated an analysis of growth polarity in a species related to Schiz. pombe. The 

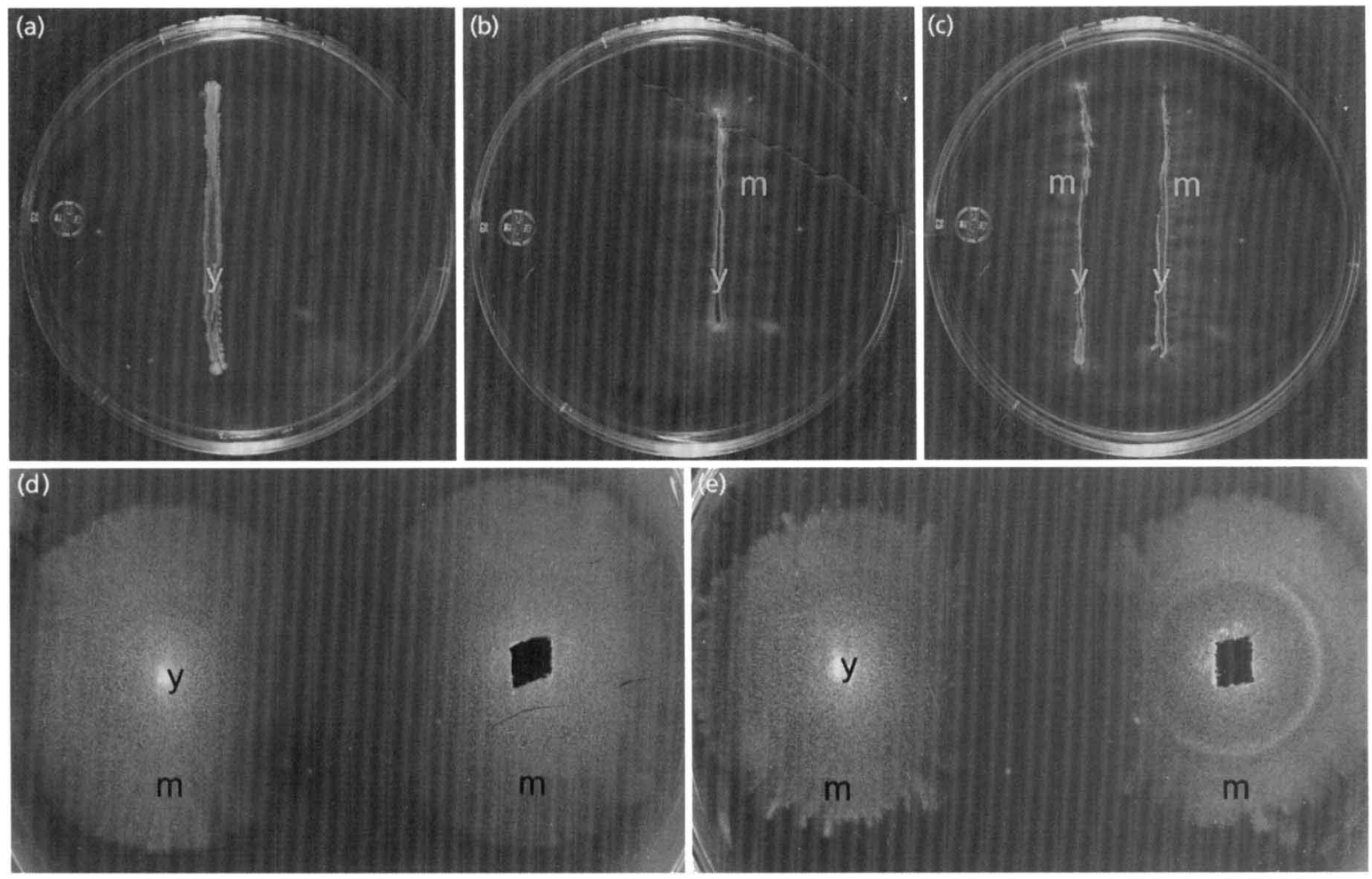

Fig. 1. Yeast-to-mycelium transition on solid medium (YEMA). (a) A 3-d-old yeast culture. (b) A 14-d-old yeast culture fringed by mycelium. (c) Progression of the mycelium towards fresh substrate. (d, e) Thickening of the mycelium caused by inverted nutrient gradient. The bright central spots in the colonies are yeasts. Holes were cut after $7 \mathrm{~d}$ incubation and filled with $20 \mu \mathrm{l} 5 \%$ glucose (d) or $20 \mu \mathrm{l} \%$ yeast extract (e). y; yeasts; m; mycelium.

fission yeasts are a group of three species (Sipiczki et al., 1982). It was noted that one species, Schiz. japonicus, occasionally developed hyphae around the yeast colonies after long periods of incubation (Yarrow, 1984). This observation provided the basis for the suggestion that present-day fission yeasts might have derived from a mycelial ancestor (Sipiczki, 1995). Here we describe the dimorphic cycle of Schiz. japonicus var. japonicus and describe certain characteristic features of the hyphal cells extending in a unipolar fashion. The distantly related budding yeast Saccharomyces cerevisiae, another frequent model for the investigation of cell polarity, can also undergo environmentally controlled morphological changes. These are due to modifications of the pattern of bud site selection and depend on the function of a MAP kinase module shared with the pheromone signalling pathway (for a review see Levin \& Errede, 1995).

\section{METHODS}

Strain and media. The fission yeast Schiz. japonicus var. japonicus Yukawa et Maki CCY-44-5-1 (CBS 354) was obtained from the Czechoslovak Collection of Yeasts (CCY), Bratislava, Slovakia. The culture media YEA, YEL, MEA and SMA were described previously (Sipiczki \& Ferenczy, 1977). YEMA and YEPA were as YEA but supplemented with $3 \%$ $(\mathrm{w} / \mathrm{v})$ malt extract and $1 \%(\mathrm{w} / \mathrm{v})$ peptone, respectively. Potato agar, corn meal agar and malt agar were prepared as described by van der Walt \& Yarrow (1984). EMM1 was a synthetic minimal medium (Mitchison, 1970). All cultures were incubated at $30^{\circ} \mathrm{C}$ unless indicated otherwise.

Time-lapse microphotography. The growth of invasive hyphae in YEA was followed and photographed using a Nikon inverted microscope. Since photographing through agar medium severely reduces resolution, thin plates $(5 \mathrm{ml}$ medium in a plastic Petri dish) were used. The growth and propagation of yeasts was photographed on the surface of YEA using an Olympus $\mathrm{BH}-2$ microscope. To reduce the disturbing optical effect of the medium, the yeast cells were plated onto a microscope slide coated with a thin film of YEA. The preparation was then covered with a coverslip and placed in a plastic Petri dish. Photographs were taken at $10-15 \mathrm{~min}$ intervals.

Fluorescence microscopy. Nuclei were stained with DAPI (4',6-diamidino-2-phenylindole) as described by Moreno et al. (1991). Actin was stained with rhodamine-conjugated phalloidin (Alfa et al., 1993); cell walls and septa were stained with calcofluor (Johnson et al., 1979). When hyphae or arthrospores were to be stained, small blocks were cut from the invasive mycelium, smashed with a glass rod and fixed. Cells were examined and photographed using an Olympus BH-2 microscope.

Electron microscopy. Small agar blocks were cut from the growing front edge of the invasive mycelium grown on thin plates $(5 \mathrm{ml}$ medium in a plastic Petri dish). The agar blocks were sandwiched between two copper discs, gently pressed to liberate hyphae from the agar and cryo-fixed by plunging into propane slush kept in liquid nitrogen. The specimens were 


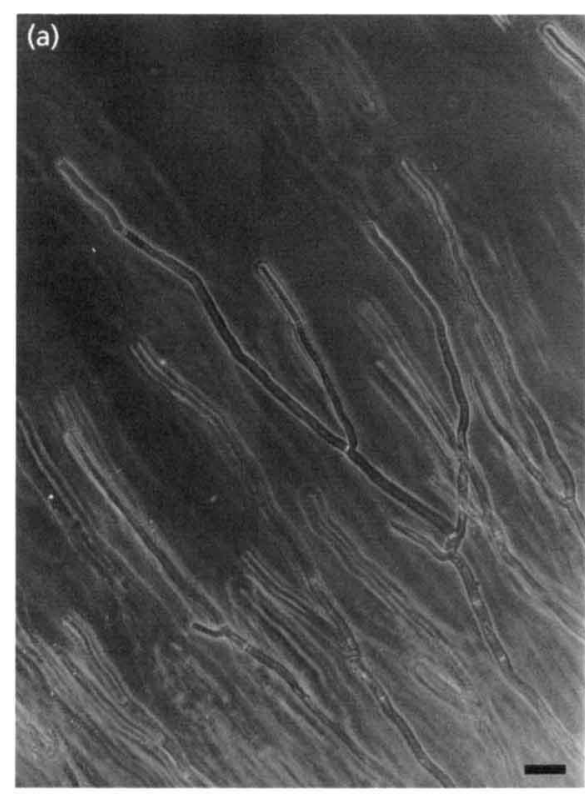

(b)

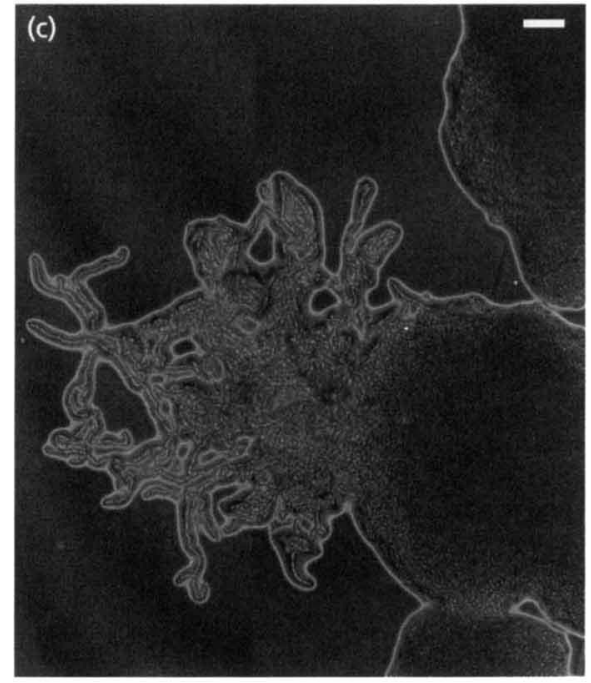

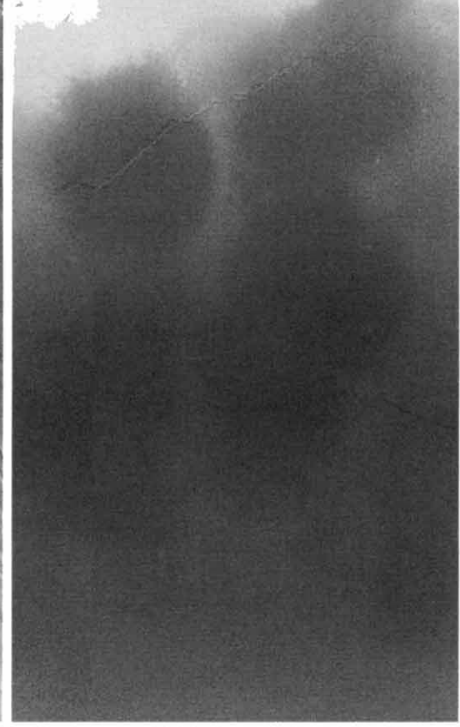

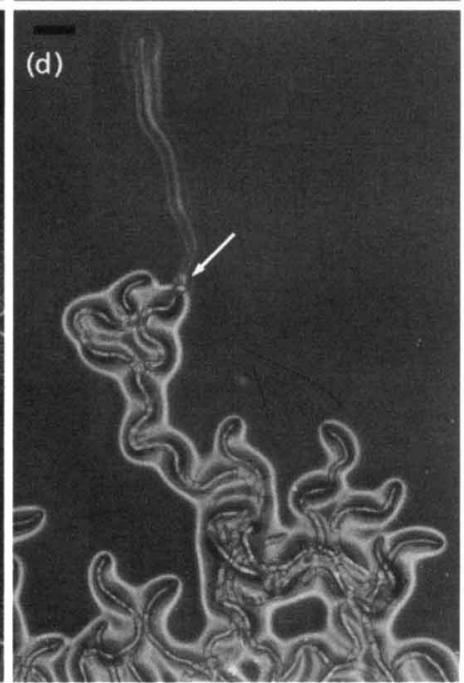

Fig. 2. Invasive and surface mycelium. (a) Invasive mycelium in YEMA. (b) The invasive mycelium grows from a few sites. This photograph was taken with an inverted microscope through the medium at low magnification. The darker patches are the area colonized by mycelium. (c) Surface mycelium on YEMA supplemented with $2 \%$ peptone. (d) A surface hypha penetrates the medium (see arrow). Bars, $25 \mu \mathrm{m}$ (a, d) or $50 \mu \mathrm{m}(\mathrm{c})$. freeze-substituted in $2 \%(\mathrm{w} / \mathrm{v})$ osmium tetroxide/acetone at $-80^{\circ} \mathrm{C}$, rinsed with acetone and embedded in epoxy resin (Yamaguchi et al., 1994). Ultrathin sections were stained with uranyl acetate and lead citrate, and observed with a JEM1200EX electron microscope (JEOL).

\section{RESULTS}

\section{Yeast-to-mycelium transition on solid substrates}

When the yeast cells of CCY-44-5-1 were inoculated onto agar media and incubated at $30^{\circ} \mathrm{C}$ they initially grew and propagated as yeast. It was only after $6-10 \mathrm{~d}$ that the first hyphae appeared around the yeast population. Once the mycelial phase was established, it rapidly developed into a contiguous mycelium that fringed the yeast colony (Fig. 1) and gradually invaded all the available substrate (note the width of the mycelial haloes in Fig. 1).

Transition to mycelial growth took place on all media tested: potato agar, corn meal agar, malt agar, YEA,
YEMA, YEPA, and the synthetic minimal media SMA and EMM1. The concentration of the components was not critical. Hyphae appeared at concentrations of $0 \cdot 25-5 \%(\mathrm{w} / \mathrm{v})$ yeast extract, $0 \cdot 25-30 \%(\mathrm{w} / \mathrm{v})$ malt extract and $0 \cdot 1-3 \%(\mathrm{w} / \mathrm{v})$ peptone, all of which allow normal growth of the yeast phase. However, when agar was omitted no hyphae appeared, suggesting that the transition required solid substrates. Furthermore, the transfer of hyphae from solid into liquid media resulted in restoration of the yeast phase.

The yeast-to-mycelium transition did not occur at all temperatures where yeasts could grow. Below $19^{\circ} \mathrm{C}$ and above $35^{\circ} \mathrm{C}$ no mycelium was formed around the growing yeasts on any medium.

\section{Nutrient gradient directs hyphal growth}

If yeasts were inoculated onto agar media as two parallel stripes, mycelia appeared only along the outside edges of the stripes (Fig. 1c). If three parallel stripes were 


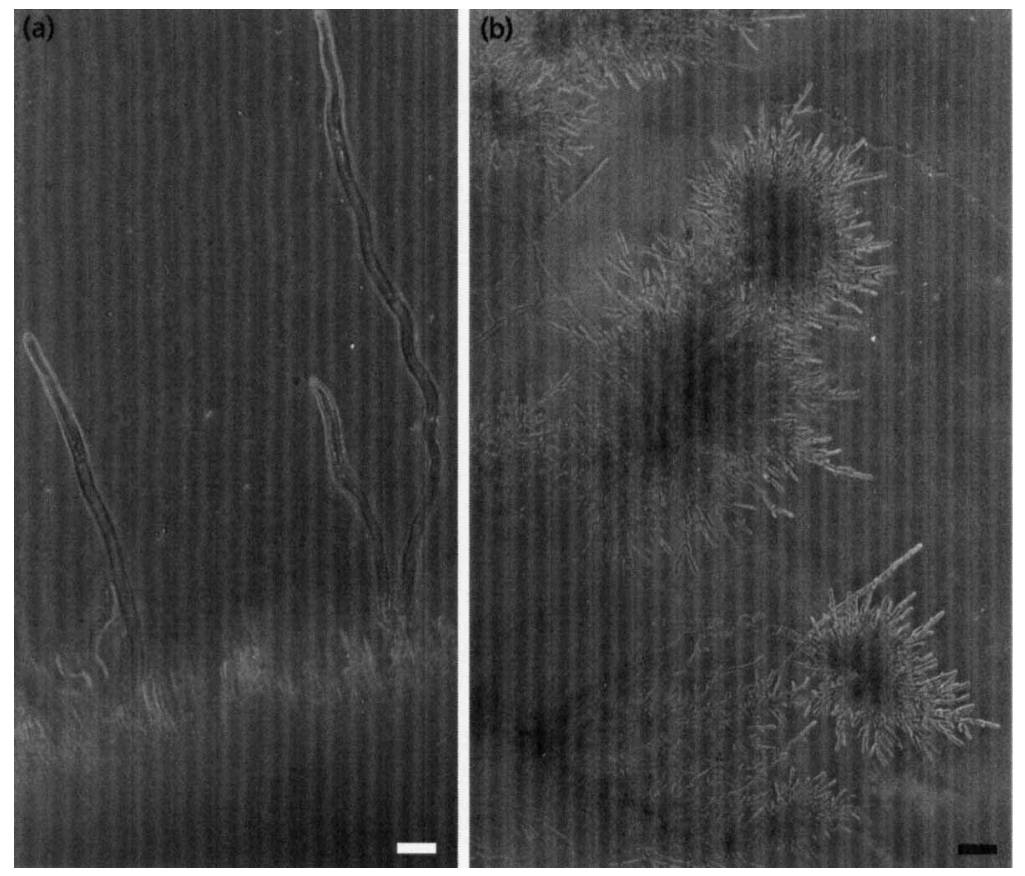

Fig. 3. Substrate invasion. (a) Front-line of the invasive mycelium with some 'fast' hyphae. (b) Satellite colonies formed by 'fast' hyphae. Bars, $25 \mu \mathrm{m}$ (a) or $100 \mu \mathrm{m}$ (b).

inoculated, the middle stripe usually did not form a mycelium. This suggests that the exhaustion of nutrients around the yeasts is not sufficient to induce the transition, which also requires the availability of fresh supplies of nutrients in the vicinity.

When the nutrient gradient was interrupted (e.g. by cutting a hole into the medium ahead of the progressing mycelium) the hyphae stopped growing before reaching the hole. If the nutrient gradient was transiently inverted by adding peptone or yeast extract solutions to holes cut behind the growing front, the mycelium transiently ceased extending. After extension resumed, a local thickening was visible behind the progressing front (see the ring in Fig. 1e), which suggests that cell growth and propagation were not halted by the inversion of the gradient. Repeated applications of nutrients into the holes at daily intervals caused several succeeding thickenings and an overall reduction in the extension of the mycelium. Glucose and malt extract did not show this effect, suggesting that it is the nitrogen gradient which directs the mycelial progression.

\section{Substrate invasion (true mycelium) and surface growth (pseudo-mycelium) in the mycelial phase}

While the yeast cells always grew on the surface of the solid media, the hyphae usually penetrated and grew into it. Since the hyphae gradually invaded large volumes of the substrate, we call them invasive. This invasive growth was characteristic on all the standard media listed in Methods. However, when we increased the concentration of yeast extract or peptone to $2 \%(\mathrm{w} / \mathrm{v})$, a mycelium growing on the surface of the medium also appeared. Fig. 2 shows the morphology of hyphae from both types of mycelium, as seen on YEMA plates. The invasive mycelium had fairly straight hyphae, in which cell separation (septum cleavage) was rare and the daughter cells remained firmly connected, which is a characteristic feature of true mycelia (van der Walt \& Yarrow, 1984). The surface hyphae showed a rather winding, almost helical morphology and a tendency to complete cytokinesis and break up into loose chains of long cells, which is typical of the pseudo-mycelium (van der Walt \& Yarrow, 1984). Occasionally some surface hyphae penetrated the surface and continued growing in the medium (Fig. 2d). Upon penetration, however, they changed their growth morphology and adopted the much straighter appearance typical of invasive hyphae. As shown in Fig. 2 (b, c) both surface and invasive mycelia grew out from a few sites. This suggests that the yeast-to-mycelium transition is rarely successful enough to establish the mycelial phase.

\section{Two strategies of substrate invasion}

The invasive mycelium formed a sharp front-line but a few hyphae usually grew ahead of the line (Fig. 3a). Time-lapse experiments revealed that the front-line progressed continuously and at a steady rate. From time-lapse photographs of five hyphae growing in YEMA at $28^{\circ} \mathrm{C}$ we calculated a mean growth rate $\left( \pm \mathrm{SE}\right.$ ) of $17 \cdot 6 \pm 2 \cdot 2 \mu \mathrm{m} \mathrm{h}^{-1}$. These hyphae divided (septated) fairly regularly, with a mean generation time of $144 \pm 21 \mathrm{~min}$. The hyphae that grew ahead of the frontline ('fast' hyphae) had periods of fast extension alternating with periods of no extension. However, even in the extension periods, their growth rate was highly variable. Thus, the mean growth rate of the fast hyphae $\left(42.07 \pm 12.3 \mu \mathrm{m} \mathrm{h}^{-1}\right)$ can be taken only as a rough 


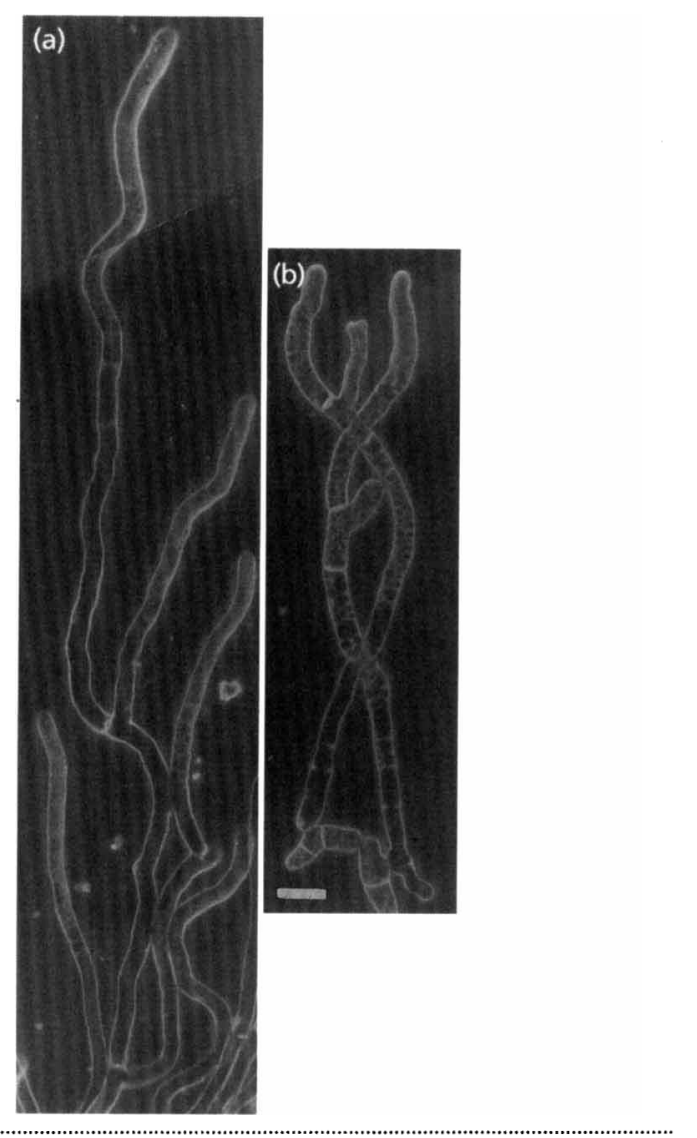

Fig. 4. Branching of hyphae. (a) Invasive hyphae. (b) Surface hyphae. Bar, $15 \mu \mathrm{m}$.

estimate. Septation was rare during extension, but it became very intense after growth had ceased. At that time the fast hypha produced several septa in rapid succession in its apical part. Shortly after the completion of septation, the resulting hyphal segments (cells) 'germinated' into new hyphae. These then grew as branches and developed a small 'satellite' colony ahead of the mycelial front-line (Fig. 3b). Upon septation, the tip of the fast hypha usually resumed fast growth and after another extension period, it underwent another serial septation, which also resulted in a new 'satellite' colony. Fig. 3 (b) shows a fast hypha that has established several satellite colonies.

\section{The mycelium shows a regular branching pattern and is composed of monokaryotic cells separated by non- perforate septa}

Both types of hyphae showed the same characteristic branching pattern (Figs $2 a, 4 a$ and $4 b$ ). The tip cells never branched. Branches were formed only at the proximal sides of the septa and developed into new hyphal tips heading towards the front edge of the mycelium. Electron microscopic examination of the septal ultrastructure revealed no pores (Fig. 5a), which suggests that the cytoplasm of adjacent cells is not connected.

To visualize nuclei, the cells were stained with DAPI (4',6-diamidino-2-phenylindole) (Fig. 6). Stationaryphase yeast cells were uninucleate, $85-90 \%$ of the exponential-phase yeast cells and $90-95 \%$ of the growing hyphal cells also contained single nuclei. Cells with two DAPI-stained bodies showed mitotic nuclear morphology.

\section{Hyphal extension is associated with extensive vacuolation and asymmetric cell divisions}

We reasoned that an important element of the efficient colonization of solid substrates could be the high speed of hyphal extension. If the hypha grows fast, it can reach fresh pools of nutrients quickly. Physical extension may not be so important in liquid media where the nutrients can diffuse more freely and the cells (yeasts) need not grow in length to reach them. We therefore compared the cell growth rate in the mycelial phase with that in the yeast phase. Using the hyphal extension rates determined by time-lapse microscopy (see above) and considering the hyphae as cylinders of $5 \mu \mathrm{m}$ diameter, we calculated the rate of cell-volume growth to be $345.4 \mu^{3} \mathrm{~h}^{-1}$ for front-line hyphae and $834.1 \mu \mathrm{m}^{3} \mathrm{~h}^{-1}$ for fast hyphae. To measure the growth rate in the yeast phase, yeast cells of exponential-phase cultures were also subjected to timelapse photography. The mean generation time $( \pm S E)$ calculated from 40 experiments was $145 \pm 19 \mathrm{~min}$. The septated cells (cells that had attained their maximum size) showed a mean size of $12.4 \pm 1.2 \mu \mathrm{m}$ long and $2 \cdot 6 \pm 0.2 \mu \mathrm{m}$ wide. Using these values and considering the yeast cells as perfect cylinders (a vast overestimation of the real size), a volume growth rate of $27 \cdot 2 \mu \mathrm{m}^{3} \mathrm{~h}^{-1}$ was calculated. This is $12-30$ times lower than the growth rate of the hyphae. This difference demonstrates that a hyphal tip produces more volume growth than the two ends of a yeast cell within the same period of time. Another possibility for the comparison of the yeast growth with the hyphal growth could be based on the determination of the specific growth rates (rate of volume increase per existing volume). In the yeast phase this value can easily be measured. In the mycelial phase, however, the highly variable cell size and the growth of the hyphae into the substrate make the determination of the exact volume rather unreliable.

How can a hypha grow faster than a yeast cell? As we examined the growth of the cells we noticed a phenomenon that could account for the faster extension in the mycelial phase. We observed that the longer hyphae usually contained large vacuoles (Fig. 7) and the formation of new septa (cell division) was followed by intense vacuolation on the proximal side of the new septa. These vacuoles expanded as the cell grew and filled a continuously increasing proportion of the total cell volume. The non-vacuolated cytoplasm rarely exceeded $40 \mu \mathrm{m}$ in length, whereas the cells could be up to several hundred micrometres long. The growth of the 

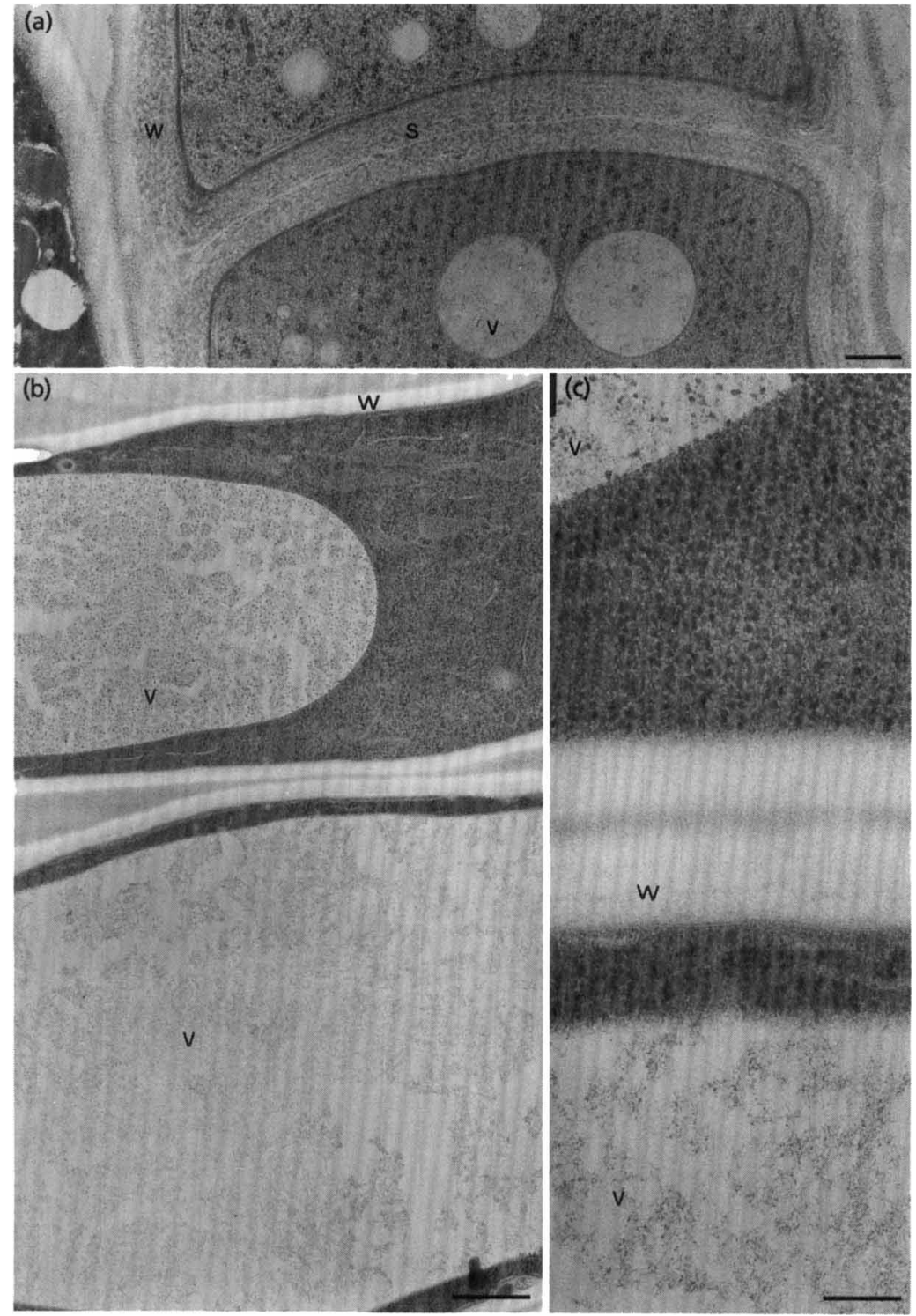

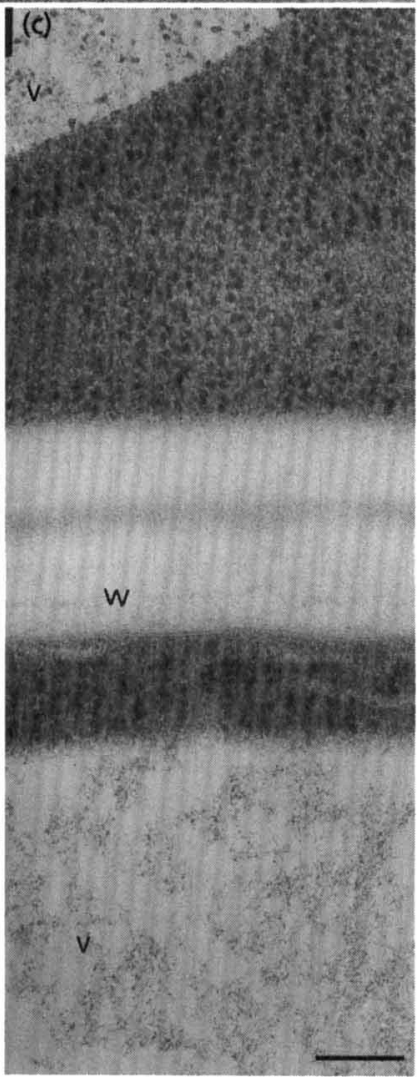

Fig. 5. Ultrastructure of hyphae. (a) Septal ultrastructure. (b, c) Vacuoles are surrounded by a thin sheet of the cytoplasm. $w$; cell wall; $s$; septum; v; vacuole. Bars, $1 \mu \mathrm{m}(\mathrm{a}, \mathrm{b})$ or $0.2 \mu \mathrm{m}(\mathrm{c})$. vacuoles probably helps the hyphae increase their volumes so fast. As long as the cell is alive, it has a continuous cytoplasm extending from pole to pole, but in the vacuolated parts this is only a thin sheet surrounding the large vacuole (Fig. $5 b, c$ ). Thus the cytoplasm itself also grows with the increasing cell volume, but it is the vacuole that fills most of the intracellular space.

Since the dense part of the cytoplasm was located apically, the hypha divided asymmetrically. The new septum was usually formed halfway between the apex and the large vacuole (see cell a in Fig. 7iii). The apical cell continued growing at its tip and soon established its own vacuoles at the sub-apical pole (cell b in Fig. 7). The sub-apical daughter cell, which inherited the large vacuoles from the mother cell, began to grow under the septum and developed into a branched hypha (cell $c$ in Fig. 7). As the cell extended, its vacuole also extended into the branch (cell $\mathrm{d}$ in Fig. 7). Its division was again asymmetric and the vacuoles were again inherited by the sub-apical daughter. This mode of growth and division resulted in very long vacuolated cells carrying as many division scars as times they had been sub-apical daughters in successive divisions (see arrows in Fig. 9a).

\section{Arthrosporogenesis in the mycelial phase}

Behind the growing front, the older parts of the mycelium usually contained yeast-like cells of heterogeneous size. We supposed that they might have arisen from hyphal fragmentation. To show that they were not yeasts, we stained them with calcofluor, a fluorescence brightener that binds predominantly to $\beta$-glucan. Calcofluor is widely used in Schiz. pombe cytology to distinguish regions of newly synthesized cell wall from regions formed from secondary septa at cell division 


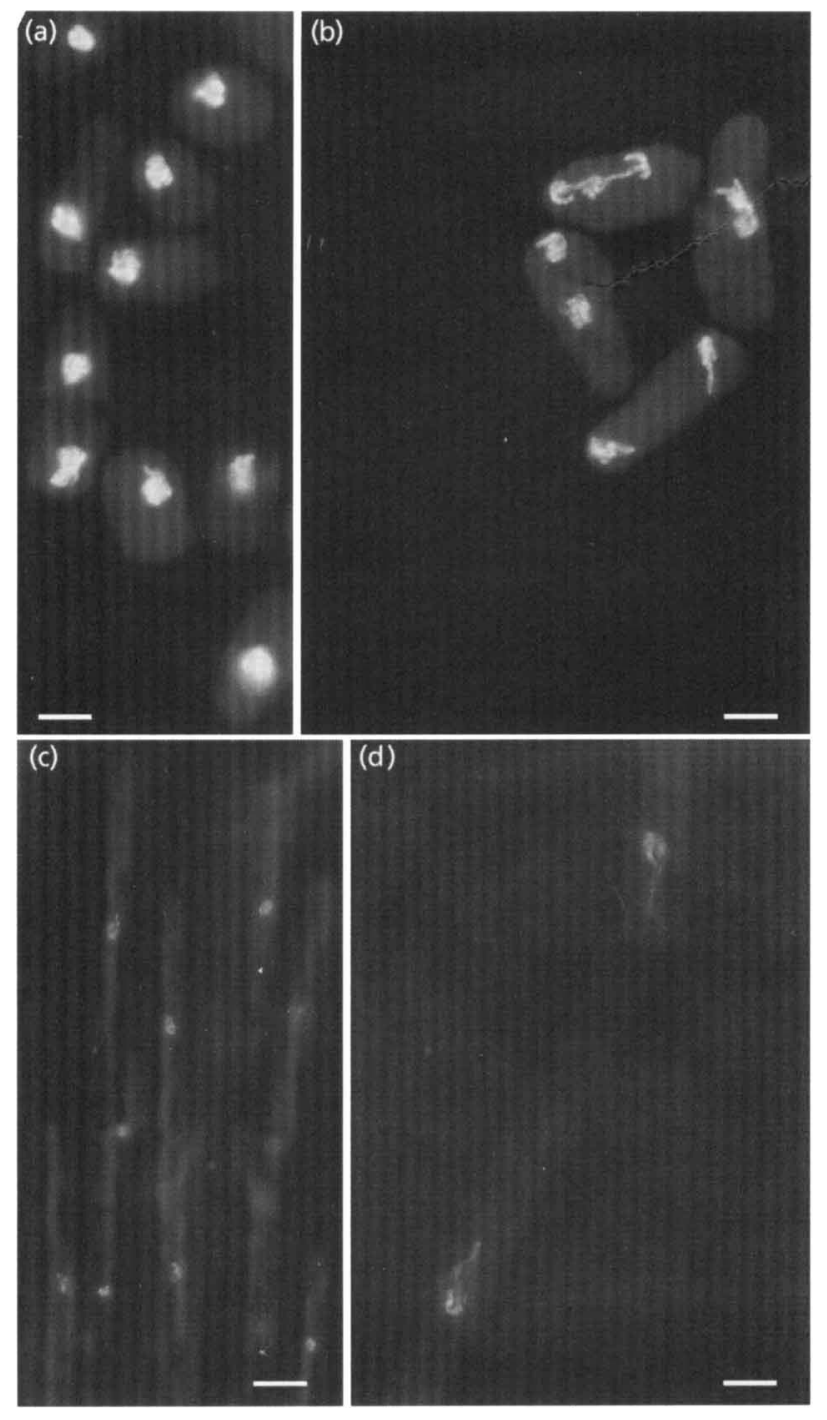

Fig. 6. DAPI-stained nuclei. (a) Interphase nuclei in yeasts. (b) Mitotic nuclei in yeasts. (c) Interphase nuclei in hyphae. (d) Mitotic nuclei in hyphae. Bars, $5 \mu \mathrm{m}(a, b, d)$ or $20 \mu \mathrm{m}$ (c).

(Johnson et al., 1979). We found that this technique can also be applied to Schiz. japonicus var. japonicus. Fig. 8 (a) shows yeast cells stained with calcofluor. By analogy with Schiz. pombe, the alternating regions can arise from cell wall synthesis (brightly staining regions) and from secondary septa (faintly staining regions). The yeast-like cells formed in the mycelium had brightly staining cylindrical middle parts and ends which stained faintly (Fig. 8c). The former may be stretches of the cell wall from the fragmenting hyphae (note the brightness of the hypha in the same figure), whereas the latter could be the secondary septa formed during septation. Thus, these cells are not yeasts but hyphal fragments, equivalents of arthroconidia (Cole, 1975). Certain hyphal fragments in Fig. 8c have not completed cytokinesis. The brightness of their septa is due to the undissolved primary septa.
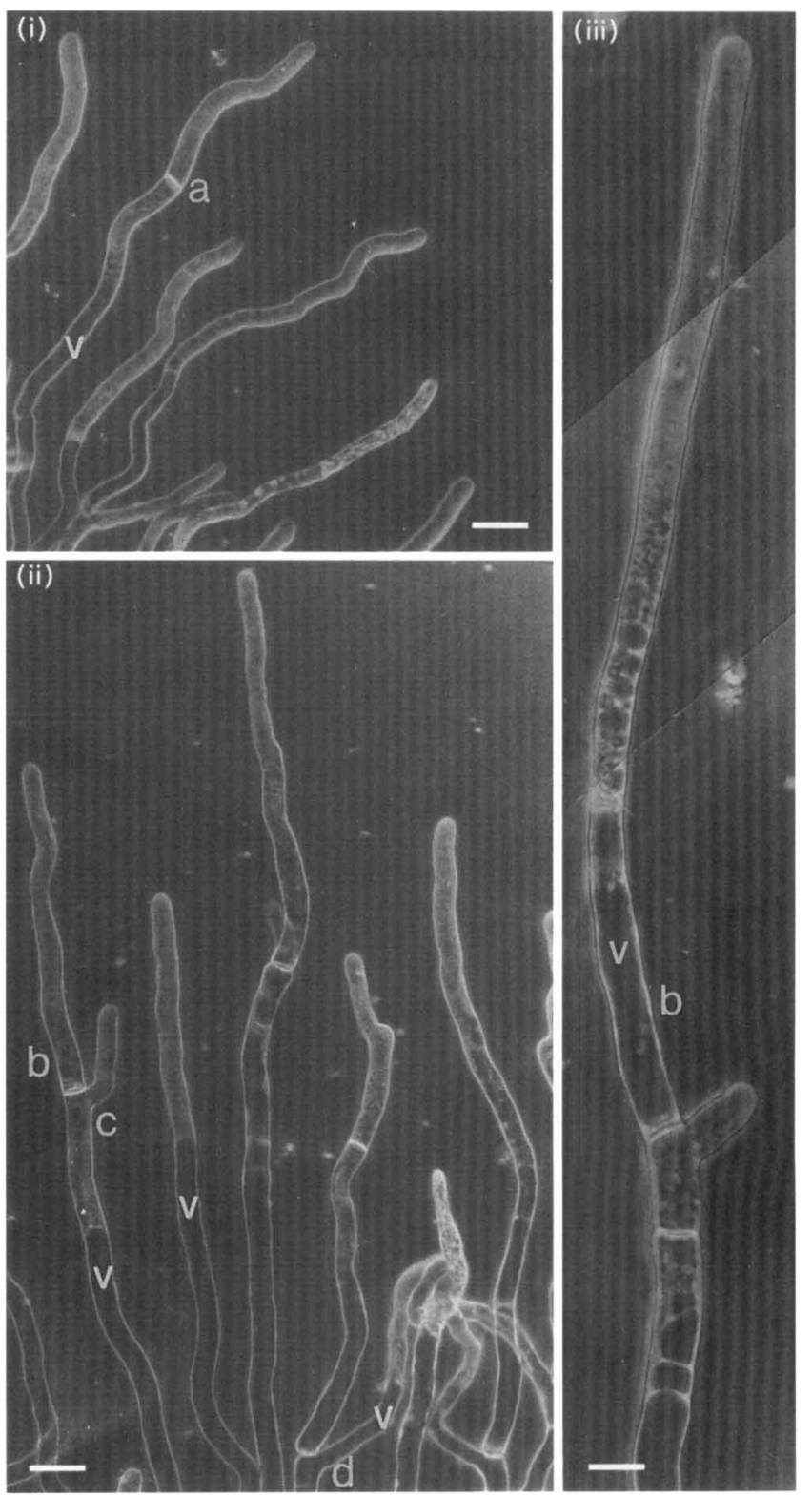

Fig. 7. Vacuolation in invasive hyphae. a, septum is formed in the middle of the non-vacuolated part of the cell; $b$, new vacuoles are formed in the apical cell after septation; c, the sub-apical daughter cell forms a new branch; $d$, the vacuole grows into the branch. $v$ : vacuolated part. Bars, $6 \mu \mathrm{m}$ (i) or $3 \mu \mathrm{m}$ (ii, iii).

\section{Shift of hyphae onto fresh medium or to high temperature causes transition to the yeast phase}

In agar media, where the yeast-to-mycelium transition is possible, the mycelium extends indefinitely as long as fresh substrate is available for invasion. As shown above, a sudden increase of the nutrient concentration in the medium holds up progression of the mycelium and elicits hyphal septation. We asked whether this process could lead to a mycelium-to-yeast transition. To test this possibility we isolated invasive hyphae, transferred them 


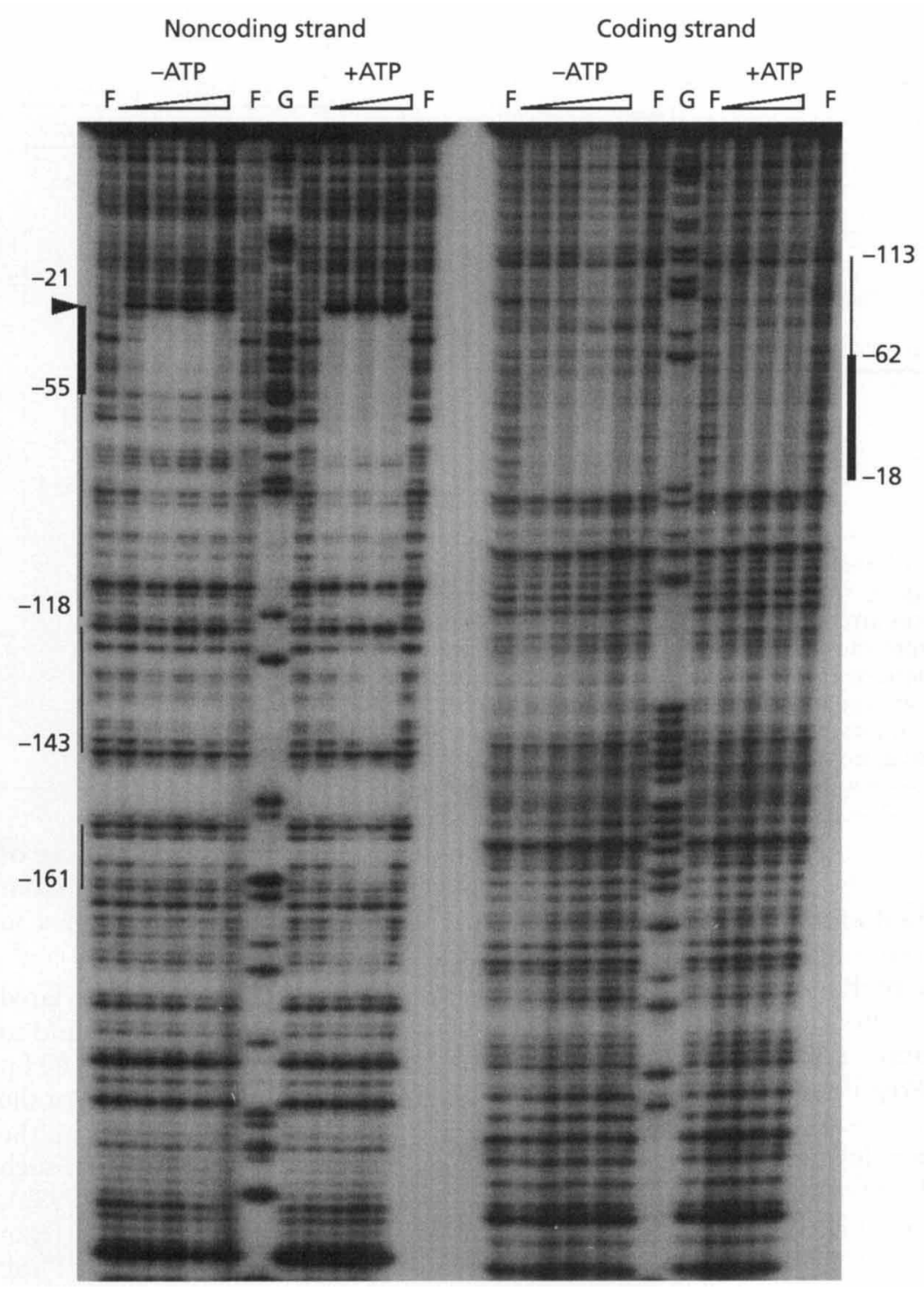

Fig. 8. Arthrosporogenesis in the mycelial phase. (a) Yeast cells, (b) fragmenting hypha and (c) free arthrospores. (a) and (c) were stained with calcofluor. $s$, septum. Bars, $2 \mu \mathrm{m}$ (a), $12 \mu \mathrm{m}$ (b) or $3 \mu \mathrm{m}$ (c).

onto the surface of fresh YEMA and covered them with microscope cover slips. Time-lapse photography (Fig. 9) revealed that the hyphae grew and underwent multiple septations. The first septum appeared after $3-4 \mathrm{~h}$ and was formed halfway between the large vacuole and the cell apex. The second septum appeared in the sub-apical daughter cell, usually after a further $60-70 \mathrm{~min}$. The third septum was formed in the apical daughter cell 10-20 min after the second. The resulting segments then began to swell and after separation (cytokinesis) also grew at their ends. The typical yeast morphology, characterized by smaller diameter and regular bipolar extension, was attained after three to four additional divisions. Cell death was frequent during this process (Fig. 9b). A return to the yeast phase could also be induced by shifting mycelial cultures to $37^{\circ} \mathrm{C}$. This temperature shift halted the extension of the hyphae and resulted in the formation of yeast microcolonies at the front edge of the mycelium.

\section{DISCUSSION}

This work demonstrates that one of the fission yeasts, Schiz. japonicus var. japonicus, is a dimorphic organism capable of producing both yeast cells and mycelia. The yeast phase is characteristic of cultures grown in liquid media but can also exist on solid media. The yeast-tomycelium transition is environmentally regulated: it requires a solid medium and a nutrient gradient.

\section{The yeast-to-mycelium transition}

A great variety of stimuli are known to induce yeast-tomycelial transitions in dimorphic fungi. These include: high $\mathrm{CO}_{2}$ tension or anaerobiosis (Mucor spp.; Bartnicki-Garcia, 1963); changes in temperature, for example in Histoplasma capsulatum (Kobayashi et al., 1985 ) and Paracoccidoides brasiliensis (San-Blas \& SanBlas, 1985); nature of the carbon source (Yarrowia 

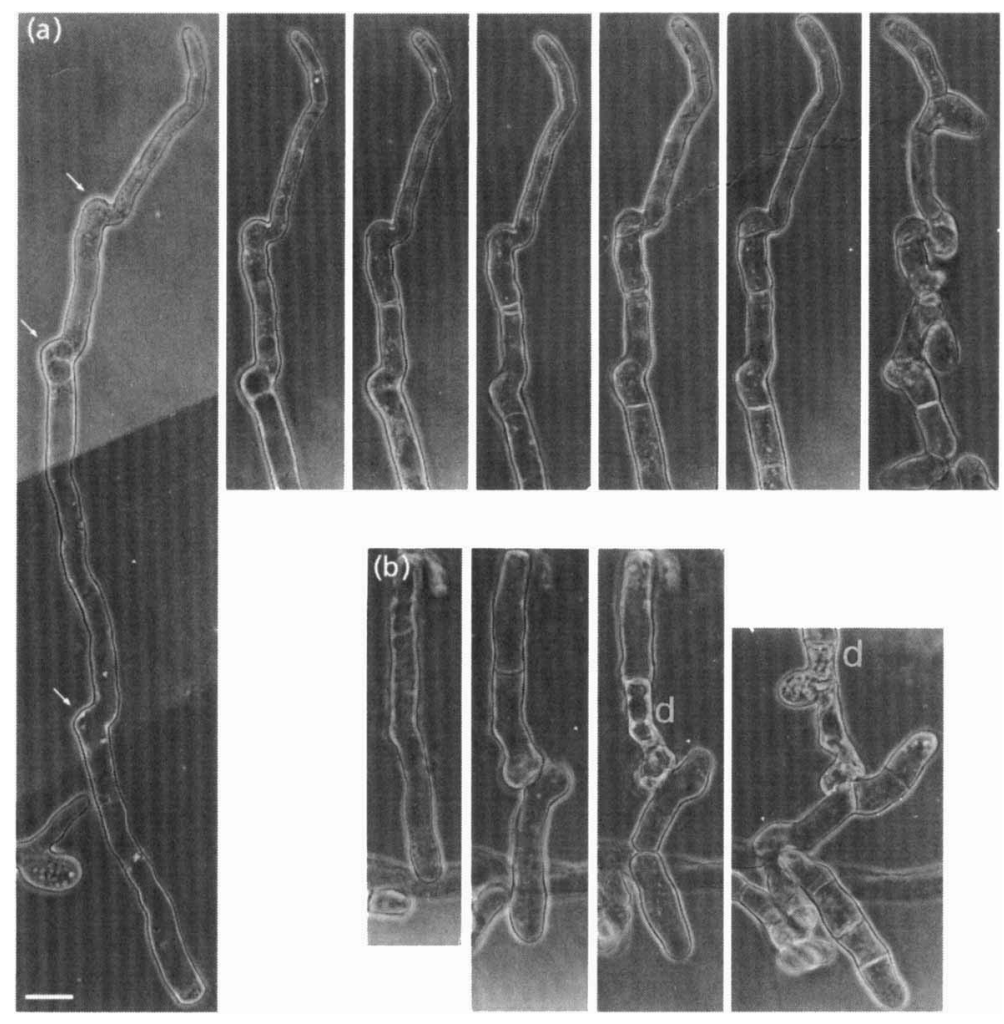

\begin{abstract}
Fig. 9. Mycelium-to-yeast transition upon shifting invasive mycelium onto fresh medium. Selected photomicrographs of a time-lapse experiment. (a) Photographs were taken at $0,80,125,230,250,330$ and $500 \mathrm{~min}$. (b) Photographs were taken at 0 , 190, 250 and 390 min. Arrows in (a) show division sites ('scars'). d, dead cell. Bar, $10 \mu \mathrm{m}$.
\end{abstract}

lipolytica; Soll, 1985); change of $\mathrm{pH}$ to neutral (Candida albicans; Soll, 1985) or acid (Ustilago maydis; RuizHerrera, 1995); a starvation period and a heat shock (Yarrowia lipolytica; Guevara-Olvera et al., 1993) and nitrogen starvation (Sacch. cerevisiae; Gimeno et al., 1992). Certain species also require a solid substrate for the switch to the mycelial phase. In Ustilago maydis mycelial growth of the dikaryotic phase can be established on solid media, and has only been achieved in liquid culture upon a transfer of cells from neutral to acid medium (Ruiz-Herrera et al., 1995). The yeast-tomycelium transition of haploid Cryptococcus neoformans is induced by nitrogen starvation on a solid surface (Wickes et al., 1996).

Apart from the solidity of the medium, the yeast-tomycelium transition in Schiz. japonicus var. japonicus is fairly insensitive to culturing conditions. It can take place on a variety of media and over a broad range of temperature and nutrient concentrations. However, it requires the availability of large amounts of uncolonized substrate in the vicinity of the yeast population. We hypothesize that the gradient of nutrients between the colonized (exhausted) and uncolonized (rich) part of the substrate induces the transition and then also directs the growth of hyphae. Robson et al. (1996) suggested that the polarized growth of Neurospora crassa hyphae may be defined by an intracellular alkaline $\mathrm{pH}$ gradient. Earlier studies demonstrated a more alkaline region of the medium around the hyphal tip of Neurospora crassa (Takeuchi et al., 1988). We found that the growth of Schiz. japonicus var. japonicus hyphae can be halted by changing, or reversing, the nitrogen-source gradient in the medium, which suggests that the $\mathrm{pH}$ of the medium may not play a major role in the process.

\section{Cellular morphology in the mycelial phase}

In the mycelial phase, three distinctive cellular morphologies can be observed: pseudo-hyphae, true hyphae and arthrospores. The pseudo-hyphae grow on the surface of the medium and are composed of loosely connected, elongated cells which show a winding, almost helical morphology. Helical hyphal growth was previously observed on the surface of agar media in Candida albicans; this was thought to be the result of the rotation of the hyphal apex as it extended to forage nutrients (Sherwood-Higham et al., 1994). The hyphae were straight in liquid media.

True mycelium is invasive: it grows into solid medium and can colonize it completely. It has septate hyphae, in which the cells are separated by complete septa. Neither calcofluor staining nor electron microscopy showed any pores or interseptal bodies characteristic of the septal structure in many filamentous ascomycetes (for a review see Garrison, 1983). This septal organization suggests that there is no intercellular cytoplasmic flow and communication in the hyphae.

The hyphae of both the pseudo-mycelium and the true mycelium show a characteristic branching pattern. In fact the cells do not branch, because growth is unipolar and always from the end located closest to the extending front of the mycelium. However, if the end of the cell is covered by an unsplit septum (which is the case in all 
cells except the tip cell of the hypha), the cell cannot grow there but, instead, establishes a growing pole at a nearby site and develops a new hyphal tip which appears as a hyphal branch. Growth inhibition by unsplit septa was also observed in a filamentous mutant of Schiz. pombe (Sipiczki et al., 1993).

Another characteristic feature of the mycelial phase is extensive vacuolation. One or a few large vacuoles fill the sub-apical part (up to $90 \%$ ) of the growing cells, so the 'dense' cytoplasm is squeezed into the growing, apical part. When the cell grows, the cytoplasm also grows, but not as fast as the remainder of the cell. Thus, it is the extension of the vacuoles that enables the hyphae to grow an order of magnitude faster than the yeasts. This is consistent with the hyphal growth model proposed by Gow \& Gooday (1987), in which the enlargement of the vacuole is one of the forces causing filamentation. Nevertheless, when comparing growth rates, one must bear in mind that the yeasts divide more frequently than the hyphal cells and each yeast division produces two new growing tips. Thus, the yeast phase may be more efficient at biomass production than the mycelial phase. If a vacuolated hyphal cell of Schiz. japonicus var. japonicus divides, the septum halves the 'dense' cytoplasm, so it is located closer to the growing cell tip. The large vacuole remains in the sub-apical daughter cell. The apical daughter does not receive visible vacuoles, but it soon develops some. The mechanism of vacuole inheritance has been studied in a variety of species and found to vary among different organisms (e.g. in Candida albicans, Gow \& Gooday, 1982; Yokoyama \& Takeo, 1983; in Sacch. cerevisiae, Hill et al., 1996). In the budding yeast Sacch. cerevisiae, the daughter cell inherits vacuole material from the mother via intracellular transport mechanisms involving actin and myosin (Hill et al., 1996). The mother cell vacuole projects tubular and vesicular structures into the bud. These vesicular structures then fuse in the new daughter cell to form one or a few larger vacuoles (Weisman \& Wickner, 1988). It can be supposed that the apical cells of the Schiz. japonicus var. japonicus hyphae do not form their vacuoles de novo either, but inherit vesicules that can be enlarged into functional vacuoles. Our attempts to stain the vacuoles with carboxyDCFDA (5-carboxy-2', $7^{\prime}$-dichlorofluorescein diacetate) or FM 4-64 (Molecular Probes) did not provide information about the origin of the hyphal vacuoles because of the very strong fluorescence background when the hyphae were stained in situ in the medium (data not shown).

\section{Mycelial growth}

Certain hyphae of the invasive mycelium grow faster than the rest. These are prone to undergo sudden, multiple septations in their non-vacuolated apical regions. Since the resulting hyphal fragments usually germinate into new hyphae, they can establish new, satellite colonies. This process might be a strategy to improve the efficiency of substrate colonization. Re- cently, a similar phenomenon was reported in Candida albicans (Radford et al., 1997), where formation of satellite colonies around the edge of the mother colony was also observed. These satellites were produced by cells that had grown deep into the agar re-emerging onto the agar surface and producing a new colony.

Multiple septation of invasive hyphae can also take place behind the growing front edge of the mycelium, but this usually results in arthrosporogenesis. Two basic modes of conidiogenesis are recognized, which are referred to as blastic and thallic (for a review see Cole $\&$ Samson, 1983). The two major forms of the thallic mode are enteroarthric development and holoarthric development. In holoarthric conidium formation, the conidia secede as a result of splitting of the septa formed in the hypha (schizolysis), and the whole wall of the fragmenting hypha is hereby retained. Schiz. japonicus var. japonicus forms conidia by simple septation and fragmentation of its hyphae. The conidia secede by the dissolution of the central (highly calcofluor-positive) layers of the septa. The rest of the septal material (calcofluor-negative) then forms the hemispherical ends of the mature conidium, whereas the wall of the fragmenting hypha provides the cylindrical (moderately calcofluor-positive) part of the wall. These features demonstrate that conidiogenesis in S. japonicus var. japonicus is a holoarthric development, resulting in true holoarthric conidia (arthroconidia).

Although the mycelium can grow as long as solid substrate is available for colonization, a sudden change of the nutritional environment or the incubation temperature can induce a return to the yeast phase. The transfer of invasive hyphae onto the surface of fresh agar medium causes an immediate cessation of tip extension and launches a process involving multiple septation in the cytoplasm of the apical part. The septa appear in a regular temporal order. The first septum halves the cytoplasm of the hyphal cell, dividing it into a longer, vacuolated sub-apical segment and a shorter apical segment. The second septum then appears in the subapical segment, always preceding the third, which appears in the apical segment. This observation suggests that the two segments differ not only in the physical size and vacuolation, but also in the duration of the cell cycle. One can speculate that the cytoplasm may contain a negative cell cycle regulator which is more abundant in the apical part. This regulator might also play a role in sustaining the fast hyphal extension by inhibiting division.

\section{Concluding remarks}

The following general picture emerges from the data presented in this paper (Fig. 10). Schiz. japonicus var. japonicus can exist in both yeast phase and mycelial phase and can develop four distinctive cellular morphologies: fission yeasts, pseudo-hyphae, true hyphae and arthrospores. The yeast phase can switch to the mycelial phase only on solid media, most probably in response to the nutritional gradient between the 


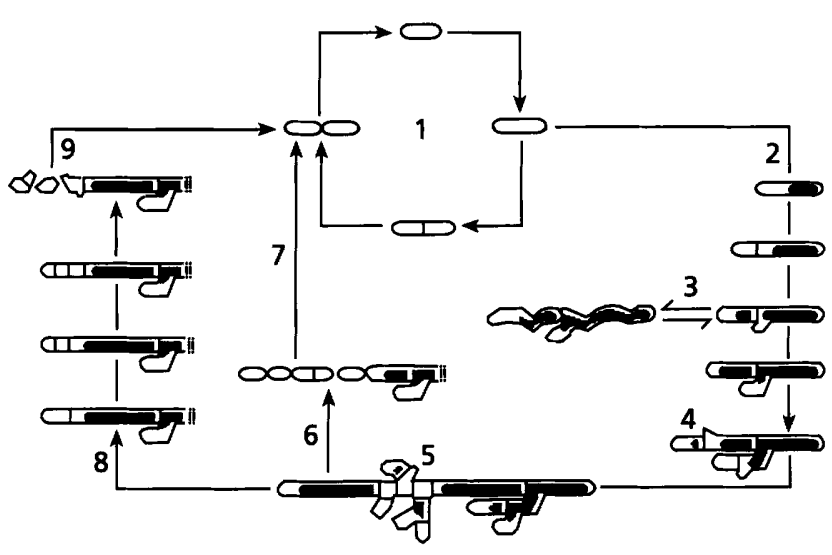

Fig. 10. The dimorphic cycle of Schiz. japonicus var. japonicus. Dark areas represent vacuoles. (1) Yeast phase. (2) Transition to unipolar growth and vacuolation. (3) Development of surfacegrowing pseudo-mycelium. (4) Development of invasive true mycelium. (5) Hyphal fragmentation and formation of a satellite colony. (6) Arthrosporogenesis. (7) Germination of arthrospores into yeasts. $(8,9)$ Mycelium-to-yeast transition.

colonized and the uncolonized regions of the substrate. This transition results in a dramatic increase of elongation rate, most probably due to the formation of large vacuoles which fill up most of the space in the hyphae. The mycelium can colonize the entire body of the substrate (true mycelium) or just its surface (pseudomycelium). In older parts of the mycelium the hyphae can also convert into arthroconidia. Elimination of the nutrient (most probably nitrogen-source) gradient induces a gradual return from the unipolar growth of the mycelial phase into the bipolar growth of the yeast phase.

What are the possible implications of these findings to the ecology of Schiz. japonicus var. japonicus on natural substrates? The morphological plasticity may ensure a better competitiveness in substrate colonization. The yeast phase is better adapted to spread through liquid substrates whereas the mycelial form is better adapted for rapid colonization of solid substrates. Thus, this remarkable organism is able to disperse by the colonization strategies of both unicellular yeasts and hyphal moulds. The morphological diversity of Schiz. japonicus var. japonicus provides a novel model for the investigation of cell polarity and morphogenesis. Its major advantage over Schiz. pombe is the ability to alternate between unipolar and bipolar growth.

\section{ACKNOWLEDGEMENTS}

We are most grateful to Dr A. Kocková-Kratochvilová for provision of the strain Schiz. japonicus var. japonicus. We thank Misako Ohkusu and Ilona Lakatos for able technical assistance. This work was supported by the Hungarian National Research Fund (OTKA) and the Ministry of Education and Culture of Hungary (MKM). M.S. is grateful to the Ministry of Education, Science and Culture of Japan for a grant which enabled him to work as a guest professor in the Research Center for Pathogenic Fungi and Microbial Toxicoses, Chiba University.

\section{REFERENCES}

Alfa, C., Fantes, P., Hyams, J., Mcleod, M. \& Warbrick, E. (1993). Experiments with Fission Yeasts. Cold Spring Harbor, NY: Cold Spring Harbor Laboratory.

Bartnicki-Garcia, S. (1963). Mold-yeast dimorphism of Mucor rouxii. Bacteriol Rev 27, 293-304.

Cole, G. T. (1975). The thallic mode of conidiogenesis in the Fungi Imperfecti. Can J Bot 53, 2983-3001.

Cole, G. T. \& Samson, R. A. (1983). Conidium and conidiospore formation in pathogenic microfungi. In Fungi Patbogenic for Humans and Animals, Part A, Biology, pp. 437-524. Edited by D. H. Howard. New York \& Basel: Marcel Dekker.

Garrison, R. G. (1983). Ultrastructural cytology of pathogenic fungi. In Fungi Pathogenic for Humans and Animals, Part A, Biology, pp. 229-321. Edited by D. H. Howard. New York \& Basel: Marcel Dekker.

Gimeno, C. J., Ljungdahl, P. O., Style, C. A. \& Fink, G. R. (1992). Unipolar cell divisions in the yeast $S$. cerevisiae lead to filamentous growth: regulation of starvation and RAS. Cell 68, 1077-1090.

Gow, N. A. R. \& Gooday, G. W. (1982). Vacuolation, branch production and linear growth of germ tubes of Candida albicans. J Gen Microbiol 128, 2195-2198.

Gow, N. A. R. \& Gooday, G. W. (1987). Cytological aspects of dimorphism in Candida albicans. Crit Rev Microbiol 15, 73-78.

Guevara-Olvera, L., Calvo-Mendez, C. \& Ruiz-Herrera, J. (1993). The role of polyamine metabolism in dimorphism of Yarrowia lipolytica. J Gen Microbiol 139, 485-493.

Hagan, I. M. \& Hyams, J. S. (1988). The use of cell division cycle mutants to investigate the control of microtubule distribution in the fission yeast Schizosaccharomyces pombe. J Cell Sci 89, 343-357.

Hill, K. L., Catlett, N. L. \& Weisman, L. S. (1996). Actin and myosin function in directed vacuole movement during cell division in Saccharomyces cerevisiae. J Cell Biol 135, 1535-1549.

Johnson, B. F., Calleja, G. B., Boisclair, I. \& Yoo, B. Y. (1979). Cell division in yeasts. III. The biassed, asymmetric location of the septum in the fission yeast cell, Schizosaccharomyces pombe. Exp Cell Res 123, 253-259.

Johnson, B. F., Calleja, G. B., Yoo, B. Y., Zuker, M. \& McDonald, I. J. (1982). Cell division: key to cellular morphogenesis in the fission yeast, Schizosaccharomyces. Int Rev Cytol 75, 167-208.

Kobayashi, G. S., Medoff, G., Maresca, B., Sacco, M. \& Kumar, B. V. (1985). Studies on phase transition in the dimorphic pathogen Histoplasma capsulatum. In Fungal Dimorphism, with Emphasis on Fungi Pathogenic for Humans, pp. 69-91. Edited by P. J. Szaniszlo. New York: Plenum.

Levin, D. E. \& Errede, B. (1995). The proliferation of MAP kinase signalling pathways in yeast. Curr Biol 7, 197-202.

Marks, J. \& Hyams, J. S. (1985). Localization of F-actin through the cell division cycle of Schizosaccharomyces pombe. Eur J Cell Biol 39, 27-32.

May, J. W. \& Mitchison, J. M. (1995). Pattern of polar extension of the cell wall in the fission yeast Schizosaccharomyces pombe. Can J Microbiol 41, 273-277.

Mitchison, J. M. (1970). Physiological and cytological methods for Schizosaccharomyces pombe. Methods Cell Physiol 4, 131-165. 
Mitchison, J. M. \& Nurse, P. (1985). Growth in cell length in the fission yeast Schizosaccharomyces pombe. J Cell Sci 75, 357-376.

Miyata, H., Miyata, M. \& Johnson, B. F. (1986). Patterns of extension growth of the fission yeast, Schizosaccharomyces pombe. Can J Microbiol 32, 528-530.

Miyata, H., Miyata, M. \& Johnson, B. F. (1990). Pattern of end growth of the fission yeast Schizosaccharomyces pombe. Can J Microbiol 36, 390-394.

Moreno, S., Klar, A. \& Nurse, P. (1991). Molecular genetic analysis of fission yeast Schizosaccharomyces pombe. Methods Enzymol 194, 795-823.

Radford, D. R., Challacombe, S. J. \& Walter, J. D. (1997). Scanning electron microscopy of the development of structured aerial mycelia and satellite colonies of phenotypically switched Candida albicans. J Med Microbiol 46, 326-332.

Robson, G. D., Prebble, E., Rickers, A., Hosking, S., Denning, D. W., Trinci, A. P. J. \& Robertson, W. (1996). Polarized growth of fungal hyphae is defined by an alkaline $\mathrm{pH}$ gradient. Fungal Genet Biol 20, 289-298.

Ruiz-Herrera, J., Leon, C. G., Guevara-Olvera, L. \& Carabez-Trejo, A. (1995). Yeast-mycelial dimorphism of haploid and diploid strains of Ustilago maydis. Microbiology 141, 695-703.

San-Blas, F. \& San-Blas, G. (1985). Paracoccidoides brasiliensis. In Fungal Dimorphism With Emphasis on Fungi Pathogenic for Humans, pp. 93-120. Edited by P. J. Szaniszlo. New York: Plenum.

Sherwood-Higham, J., Zhu, W.-Y., Devine, C. A., Gooday, G. W., Gow, N. A. R. \& Gregory, D. W. (1994). Helical growth of hyphae of Candida albicans. J Med Vet Mycol 32, 437-445.

Sipiczki, M. (1995). Phylogenesis of fission yeasts. Contradictions surrounding the origin of a century old genus. Antonie Leeuwenboek 68, 119-149.

Sipiczki, M. \& Ferenczy, L. (1977). Protoplast fusion of Schizosaccharomyces pombe auxotrophic mutants of identical matingtype. Mol Gen Genet 151, 77-81.

Sipiczki, M. \& Grallert, A. (1997). Polarity, spatial organisation of cytoskeleton, and nuclear division in morphologically altered cells of Schizosaccharomyces pombe. Can J Microbiol 43, 991-998.

Sipiczki, M., Kucsera, J., Ulaszevski, S. \& Zsolt, J. (1982).
Hybridization studies by crossing and protoplast fusion within the genus Schizosaccharomyces Lindner. J Gen Microbiol 128, 1989-2000.

Sipiczki, M., Grallert, B. \& Miklos, I. (1993). Mycelial and syncytial growth in Schizosaccharomyces pombe induced by novel septation mutations. J Cell Sci 104, 485-493.

Snell, V. \& Nurse, P. (1993). Investigations into the control of cell form and polarity: the use of morphological mutants in fission yeast. Development 1993 Supplement 289-299.

Soll, D. R. (1985). Candida albicans. In Fungal Dimorphism with Emphasis on Fungi Pathogenic for Humans, pp. 167-195. Edited by P. J. Szaniszlo. New York: Plenum.

Tekeuchi, Y., Schmid, J., Caldwell, J. H. \& Harold, F. (1988). Transcellular ion currents and extension of Neurospora crassa. J Membr Biol 101, 33-41.

van der Walt, J. P. \& Yarrow, D. (1984). Methods for the isolation, maintenance, classification and identification of yeasts. In The Yeasts. A Taxonomic Study, pp. 45-104. Edited by N. J. W. Kreger-van Rij. Amsterdam: Elsevier.

Weisman, L. S. \& Wickner, W. T. (1988). Intervacuolar exchange in the yeast zygote: a new pathway in organelle communication. Science 241, 589-591.

Wickes, B. L., Mayorga, M. E., Edman, U. \& Edman, J. C. (1996) Dimorphism and haploid fruiting in Cryptococcus neoformans: association with the $\alpha$-mating type. Proc Natl Acad Sci USA 93, 7327-7331.

Yamaguchi, M., Miyatsu, Y., Horikawa, Y., Sugahara, K., Mizokami, H., Kawase, M. \& Tanake, H. (1994). Dynamics of hepatitis $B$ virus core antigen in a transformed yeast cell: analysis with an inducible system. J Electron Microsc 43, 386-393.

Yarrow, D. (1984). Schizosaccharomyces Lindner. In The Yeasts. A Taxonomic Study, pp. 414-422. Edited by N. J. W. Kreger-van Rij. Amsterdam: Elsevier.

Yokoyama, K. \& Takeo, K. (1983). Differences of asymmetrical division between the pseudomycelial and yeast forms of Candida albicans and their effect on multiplication. Arch Microbiol 134, 251-253.

Received 23 September 1997; revised 19 November 1997; accepted 14 January 1998. 